\title{
Reviewers - Volume 23
}

$\begin{array}{ll}\text { Nora Bacon } & \text { University of Nebraska } \\ \text { Omaha } \\ \text { Rick Battistoni } & \text { Providence College } \\ \text { Robert Bringle } & \begin{array}{l}\text { Indiana University Purdue } \\ \text { University at Indianpolis }\end{array} \\ \text { Susan Cashman } & \text { University of Massachusetts } \\ \text { Beth Catlett } & \text { DePaul University } \\ \text { Patti Clayton } & \text { PHC Ventures } \\ \text { David Cooper } & \text { Michigan State University } \\ \text { Thomas Deans } & \text { University of Connecticut } \\ \text { Lina Dostilio } & \text { Duquesne University } \\ \text { Michelle Dunlap } & \text { Connecticut College } \\ \text { Thomas Ehrlich } & \text { Stanford University } \\ \text { Sandra Enos } & \text { Bryant University } \\ \text { Joseph Erickson } & \text { Augsburg College } \\ \text { Janet Eyler } & \text { Vanderbilt University } \\ \text { Peter Felton } & \text { Elon University } \\ \text { Jessica Fogel } & \text { University of Michigan } \\ \text { Andy Furco } & \text { University of Minnesota } \\ \text { Sherril Gelmon } & \text { Portland State University } \\ \text { David Greene } & \text { Colorado State University } \\ \text { Thomas Hahn } & \text { Indiana University } \\ & \text { Purdue University at } \\ \text { Indianapolis } \\ \text { Susan Harris } & \text { University of Southern } \\ \text { Eric Hartman } & \text { California } \\ \text { Julie Hatcher } & \text { Kansas State University } \\ \text { Barbara Holland } & \text { University at Indianapolis } \\ \text { Barbara Jacoby } & \text { Independent Consultant } \\ \text { Rob Jagers } & \text { University of Maryland } \\ \text { Emily Janke } & \text { University of Michigan } \\ \text { Susan Jones } & \text { University of North Carolina } \\ \text { Gathy Jordan } & \text { Greensboro } \\ \text { The Ohio State University } \\ \text { University of Minnesota } \\ \text { Mills College }\end{array}$

$\begin{array}{ll}\text { Richard Kendrick } & \text { SUNY - Cortland } \\ \text { Cheryl Keen } & \text { Walden University } \\ \text { Arthur Keene } & \text { University of } \\ & \text { Massachusetts Amherst } \\ \text { Novella Keith } & \text { Temple University } \\ \text { Darcy Lear } & \text { Independent Scholar } \\ \text { Kelly Lockeman } & \text { Virginia Commonwealth } \\ & \text { University }\end{array}$

Kathleen Maas Weigert Loyola University Chicago Clark Maddux Appalachian State University

Sam Marullo Wesley Seminary

Katerhine Mead Independent Scholar

Ellen Middaugh San Jose State University

A.T. Miller Cornell University

Jerry Miller University of Michigan

Tania Mitchell University of Minnesota

Barbara Moely Tulane University

David Moore New York University

Keith Morton Providence College

Kerry Ann O’Meara University of Maryland

Andrew Pearl University of North Georgia

Lane Perry Western Carolina University

Kenneth Reardon Memphis University

Roger Reeb University of Dayton

Nora Pillard Reynolds Temple University

John Saltmarsh University of

Massachusetts Boston

Lorilee Sandmann University of Georgia

Rob Shumer University of Minnesota

Timothy Stanton Stanford University

Randy Stoecker University of Wisconsin

Robert Swap University of Virginia

Kelly Ward Washington State University

Kathleen Maas Weigert Loyola University Chicago

Edward Zlotkowski Bentley University 